\title{
WhatsAPP in neurosurgery: the best practice is in our hands
}

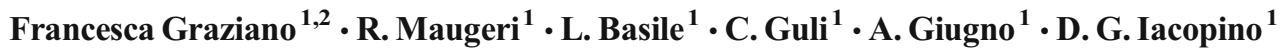

Received: 10 January 2017 / Accepted: 16 January 2017 / Published online: 1 February 2017

(C) Springer-Verlag Wien 2017

\section{Dear Sir,}

We are very pleased that our letter found interest in the neurosurgical community, as comments following our letter have demonstrated. The considerations expressed by Shaller and Anderson et al. emphasize that the concept of virtual communication along with its implications is a crucial and delicate topic to manage.

As highlighted by Anderson, a balance needs to be struck between the potential benefits of a new technology and concerns regarding data transfer security [1-3]. Regarding this, we would add potentially reassuring information.

The Italian Data Protection Authority (DPA) is an important legal authority aiming to protect individual privacy and data transfer security. WhatsApp is actually under their scrutiny to assure privacy maintenance. In September 2016, the DPA investigated the changes in WhatsApp's privacy policy made at the end of August to enable passing on information on WhatsApp users' accounts to Facebook for purposes including marketing.

The DPA requested WhatsApp and Facebook to provide information in order to assess the case thoroughly. Thus, it has to be acknowledged that a step forward has already been taken, applying end-to-end cryptography to WhatsApp messages.
We believe that the end-to-end technology has enhanced the security of data transfer and represents one of the important changes necessary to protect individual privacy while assuring fast, easy and "life-saving" communication.

Considering the vital importance of technology in our daily medical lives, software industries should be encouraged to elaborate novel systems to enhance data transfer security.

\section{Compliance with ethical standards}

Conflict of interest The authors declare that they have no conflicts of interest. No funds were received in support of this work.

\section{References}

1. Anderson IA, Goodden JR (2017) Editorial Re: WhatsAPP in neurosurgery: the best practice is in our hands. Acta Neurochir

2. Graziano F, Maugeri R, Giugno A, Iacopino DG (2016) WhatsAPP in neurosurgery: the best practice is in our hands. Acta Neurochir 158:2173-2174

3. Schaller K (2016) Editorial re: WhatsAPP in neurosurgery: the best practice is in our hands. Acta Neurochir 158:2175

Francesca Graziano

Francesca.graziano03@unipa.it; franeurosurgery@libero.it

1 Department of Experimental Biomedicine and Clinical

Neurosciences, Neurosurgical Clinic, AOPU "P. Giaccone"

University Study of Palermo, Palermo, Italy

2 Department of Experimental Biomedicine and Clinical Neurosciences, School of Medicine, Neurosurgical Clinic, University of Palermo, Via del Vespro 129, 90100 Palermo, Italy 